\title{
EL CARIOTIPO DE ANADENANTHERA COLUBRINA VAR. CEBIL Y DE PARAPIPTADENIA RIGIDA (LEGUMINOSAE- MIMOSOIDEAE)
}

por ANA I. HONFI y JULIO R. DAVIÑA'

\section{Summary}

The somatic chromosomes of two tree species have been studied, both of them have $2 n=26$. The karyotypes of Anadenanthera colubrina (Vell.) Brenan var. cebil (Griseb.) Altschul (18m $+8 \mathrm{sm})$ and Parapiptadenia rigida (Benth.) Brenan $(12 \mathrm{~m}+14 \mathrm{sm})$ are described for the first time. These species may be distinguished by karyotype formula, and total chromosome length.

\section{Introducción}

Los géneros Parapiptadenıa Brenan y Anadenanthera Speg. comprenden respectivamente dos y cuatro especies de América tropical y subtropical, (Burkart, 1972). P. rigida (Benth.) Brenan (=Piptadenia rigida Benth.), conocida vulgarmente como "anchico colorado" es una especie propia del Sur de Brasil, Paraguay y NE de Argentina donde se la encuentra en el estrato arbóreo de la selva de Misiones y selvas en galería del NE de Corrientes (Castiglioni, 1979). Por su parte, A. colubrina (Vell.) Brenan var. cebil (Griseb.) Altschul =Piptadenia macrocarpa Benth.; Acacia cebil Griseb.), es el conocido "curupay", " cebil colorado" o "cebil" de Jujuy, Salta, Tucumán, Catamarca, Santiago del Estero, Misiones y Corrientes (Digilio y Legname, 1966 sub. Piptadenia macrocarpa Benth.) y Córdoba (Hunziker, 1973).

La posición sistemática de estas especies ha sido discutida y tratada por varios autores. Las especies estudiadas pertenecieron al género Piptadenia Benth. y posteriormente fueron excluidas del mismo e incorporadas a géneros distintos debido a sus diferencias morfológicas

1 Proyecto de Citogenética Vegetal, Facultad de Ciencias Exactas, Cuímicas y Naturaies - U.Na.M. Rivadavia 2370 -(3300) Posadas - Misiones, Argentina.
(Spegazzini, 1922; Burkart, 1943, 1949, 1969 y 1972; Brenan, 1955 y 1963; Cowan \& Brenan, 1960; Altschul, 1964; entre otros).

En cuanto a la citología de estos taxones, existen escasos antecedentes. Goldblatt (1981) realizó el primer recuento de $2 \mathrm{n}=26$ cromosomas para $P$. rigida y Gibbs e Ingram (1982) publicaron un recuento cromosómico de $2 n=26$ para A. colubrina (Vell.) Brenan. En este trabajo se presentan y discuten por primera vez los cariotipos de $P$. rigida y $A$. colubrina var. cebil.

\section{Material y Métodos}

Las especies estudiadas se coleccionaron en Misiones, Argentina, y los ejemplares testigo son: P. rigida, Garupá, Cardozo 272, 28-VI-1996 (MNES) y A. colubrina var. cebil, Posadas, cult. Dematteis y Rodríguez 03, 21-VII-1992 (MNES, CTES); Dematteis 25, 29-X-1992 (MNES, CTES).

Los cromosomas mitóticos fueron estudiados en ápices de raicillas obtenidas de semillas germinadas, pretratadas con 8-hidroxiquinoleína $0,002 \mathrm{M}$ durante $3 \mathrm{hs}$. a $22^{\circ} \mathrm{C}$. posteriormente fijadas en etanol: ácido acético (3:1). Para la coloración se utilizó la técnica de Feulgen. Los meristemas se disgregaron y aplastaron sobre una gota de orceína lactopropiónica al $2 \%$. 
La descripción del cariotipo se realizó de acuerdo con la nomenclatura propuesta por Levan et al. (1964). Los respectivos idiogramas se confeccionaron a partir de dibujos hechos con cámara clara de 10 placas metafásicas y se basaron en los valores medios calculados para cada especie. Para determinar la posición del centrómero de cada cromosoma se estimó el índice centromérico (i= s/c x100) y los cromosomas se clasificaron en metacéntricos $(\mathrm{m})$ i =50-37,5 y submetacéntricos $(\mathrm{sm}) \mathrm{i}=37,5-25$.

La asimetría cariotípica fue analizada utilizando las categorías de Stebbins (1971) y los índices de asimetría intracromosómica (A1) e intercromosómica (A2) de Romero Zarco (1986).

\section{Resultados y Discusión}

En Anadenanthera colubrina var. cebil y Parapiptadenia rigida se observaron $2 \mathrm{n}=26 \mathrm{cro}-$ mosomas y estos resultados confirman los recuentos cromosómicos previos realizados por Gibbs e Ingram (1982) y Goldblatt (1981).

El cariotipo de $A$. colubrina está constituido por $18 \mathrm{~m}+8 \mathrm{sm}$ (Figs. 1A y 2A). Los 9 pares metacéntricos presentan longitudes entre 1,94
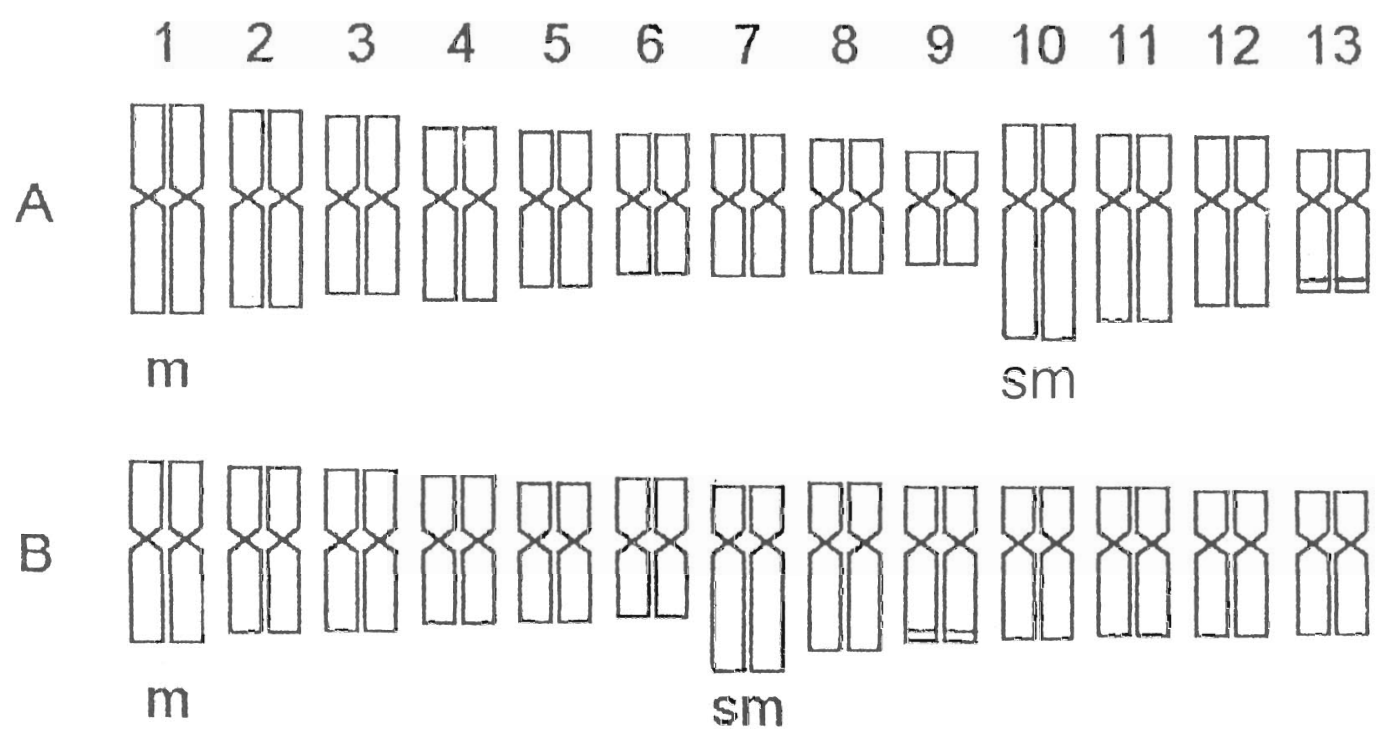

Fig. 1. Idiogramas: A, Anadenanthera colubrina var. cebil $(18 \mathrm{~m}+8 \mathrm{sm})$ y B, Parapiptadenia rigida $(12 \mathrm{~m}+14 \mathrm{sm})$. La barra representa 5 micras.
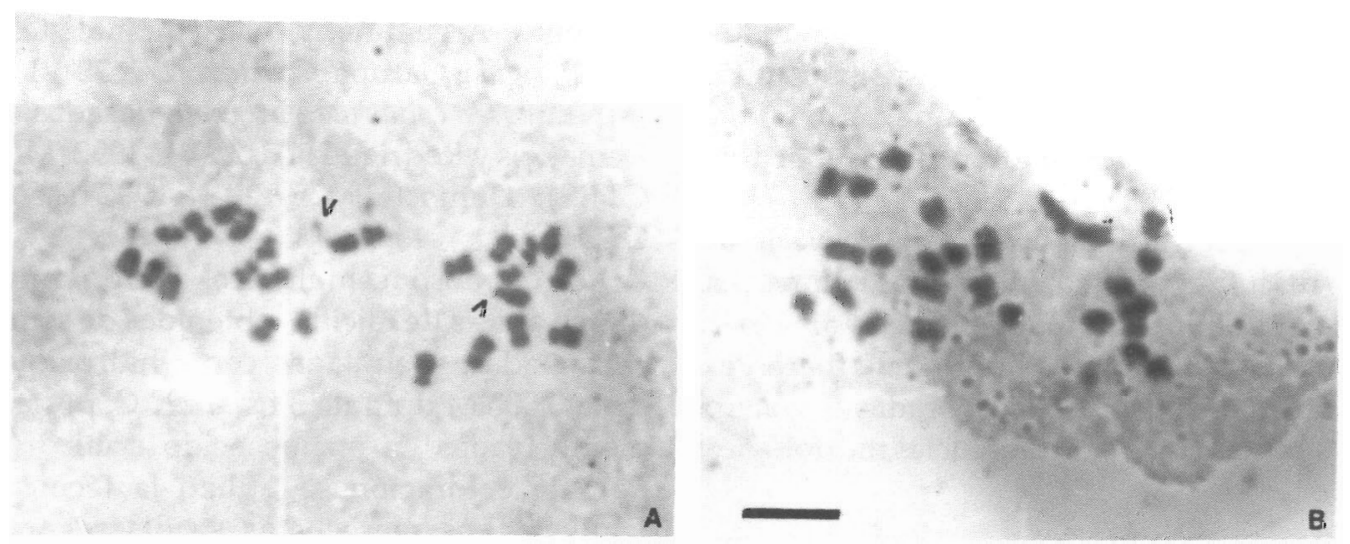

Fig. 2. Metafases mitóticas : A, Anadenanthera colubrina var. cebil $2 \mathrm{n}=26$ y $\mathrm{B}$, Parapiptadenia rigida $2 \mathrm{n}=26$. La flecha indica el satélite. La barra representa 10 micras. 
y 0,93 micras y los 4 pares submetacéntricos restantes entre 2,01 y 1,26 micras (Tabla 1 ).

Por su parte, $P$. rigida presenta un cariotipo formado por $12 \mathrm{~m}+14 \mathrm{sm}$ (Figs. 1B y 2B). Las longitudes cromosómicas varían de 1,67 a 1,22 micras en los pares metacéntricos y de 1,72 a 1,27 micras en los submetacéntricos (Tabla 2). Las longitudes máximas en ambas especies se encuentran en los cromosomas submetacéntricos y las menores se observan entre los cromosomas metacéntricos.
Ambas especies difieren en su respectiva fórmula cariotípica, A. colubrina presenta menor cantidad de pares submetacéntricos que $P$. rigida. Además en esta se observó en el brazo largo del par 9 un satélite de 0,5 micras de longitud y en $A$. colubrina en el brazo largo del par 13 (Fig. 2A y Tabla 3).

El índice centromérico medio estimado en A. colubrina es de 40,33; en cambio, en $P$. rigida es de 37,58 . También se observaron diferencias en cuanto a la longitud total del complemento,

Tabla 1. Medidas cromosómicas expresadas en $\mu \mathrm{m}$, razón l/s ( $\mathrm{r}$ ) e índice centromérico (i) de A. colubrina var. cebil. $\mathrm{s}=$ brazo corto; $\mathrm{l}=$ brazo largo; $\mathrm{c}=$ longitud total.

\begin{tabular}{|c|c|c|c|c|c|c|c|}
\hline \multirow[t]{2}{*}{ Par } & \multicolumn{3}{|c|}{$\begin{array}{c}\text { Longitudes cromosómicas medias } \\
\pm \text { error estándar }\end{array}$} & \multirow[t]{2}{*}{$\mathrm{i}$} & \multirow[t]{2}{*}{$r$} & \multirow[t]{2}{*}{$\begin{array}{l}\text { Longitud } \\
\text { relativa }\end{array}$} & \multirow[t]{2}{*}{ Tipo } \\
\hline & 3 & 1 & C & & & & \\
\hline 1 & $0,84 \pm 0,047$ & $1,10 \pm 0,101$ & $1,94 \pm 0,149$ & 43,29 & 1,31 & 10,3 & $\mathrm{~m}$ \\
\hline 2 & $0,79 \pm 0,046$ & $1,04 \pm 0,054$ & $1,83 \pm 0,099$ & 43,16 & 1,31 & 9,4 & $\mathrm{~m}$ \\
\hline 3 & $0,74 \pm 0,015$ & $0,88 \pm 0,059$ & $1,63 \pm 0,045$ & 45,39 & 1,18 & 8,4 & $\mathrm{~m}$ \\
\hline 4 & $0,62 \pm 0,013$ & $0,94 \pm 0,032$ & $1,57 \pm 0,026$ & 39,49 & 1,51 & 8,1 & $\mathrm{~m}$ \\
\hline 5 & $0,58 \pm 0,010$ & $0,80 \pm 0,013$ & $1,39 \pm 0,022$ & 41,72 & 1,37 & 7,1 & $\mathrm{~m}$ \\
\hline 6 & $0,57 \pm 0,000$ & $0,67 \pm 0,045$ & $1,24 \pm 0,045$ & 45,96 & 1,17 & 6,4 & $\mathrm{~m}$ \\
\hline 7 & $0,57 \pm 0,000$ & $0,68 \pm 0,068$ & $1,25 \pm 0,068$ & 45,60 & 1,19 & 6,1 & $\mathrm{~m}$ \\
\hline 8 & $0,51 \pm 0,040$ & $0,64 \pm 0,047$ & $1,15 \pm 0,085$ & 44,34 & 1,25 & 5,6 & $\mathrm{~m}$ \\
\hline 9 & $0,38 \pm 0,000$ & $0,55 \pm 0,013$ & $0,93 \pm 0,013$ & 40,86 & 1,44 & 5 & $\mathrm{~m}$ \\
\hline 10 & $0,67 \pm 0,045$ & $1,34 \pm 0,045$ & $2,01 \pm 0,090$ & 33,33 & 2,00 & 10,3 & $\mathrm{sm}$ \\
\hline 11 & $0,57 \pm 0,000$ & $1,14 \pm 0,010$ & $1,71 \pm 0,010$ & 33,33 & 2,00 & 8,8 & $\mathrm{sm}$ \\
\hline 12 & $0,54 \pm 0,020$ & $0,99 \pm 0,020$ & $1,53 \pm 0,000$ & 35,29 & 1,83 & 8 & $\mathrm{sm}$ \\
\hline 13 & $0,41 \pm 0,026$ & $0,84 \pm 0,032$ & $1,26 \pm 0,046$ & 32,53 & 2,04 & 6,5 & $\mathrm{sm}$ \\
\hline
\end{tabular}

Tabla 2. Medidas cromosómicas expresadas en $\mu \mathrm{m}$, razón l/s (r) e índice centromérico (i) de P. rigida. $\mathrm{s}=$ brazo corto; $\mathrm{l}=$ brazo largo; $\mathrm{c}=$ longitud total .

\begin{tabular}{|c|c|c|c|c|c|c|c|}
\hline \multirow[t]{2}{*}{ Par } & \multicolumn{3}{|c|}{$\begin{array}{l}\text { Longitudes cromosómicas medias } \\
\pm \text { error estándar }\end{array}$} & \multirow[t]{2}{*}{$\mathrm{i}$} & \multirow[t]{2}{*}{$\mathrm{r}$} & \multirow[t]{2}{*}{$\begin{array}{l}\text { Longitud } \\
\text { relativa }\end{array}$} & \multirow[t]{2}{*}{ Tipo } \\
\hline & $s$ & 1 & c & & & & \\
\hline 1 & $0,69 \pm 0,062$ & $0,98 \pm 0,039$ & $1,67 \pm 0,079$ & 41,31 & 1,42 & 9 & $\mathrm{~m}$ \\
\hline 2 & $0,63 \pm 0,039$ & $0,85 \pm 0,043$ & $1,49 \pm 0,041$ & 42,28 & 1,34 & 7,9 & $\mathrm{~m}$ \\
\hline 3 & $0,62 \pm 0,041$ & $0,84 \pm 0,052$ & $1,46 \pm 0,070$ & 42,46 & 1,35 & 7,8 & $\mathrm{~m}$ \\
\hline 4 & $0,57 \pm 0,000$ & $0,74 \pm 0,052$ & $1,32 \pm 0,052$ & 43,18 & 1,29 & 7 & $\mathrm{~m}$ \\
\hline 5 & $0,50 \pm 0,039$ & $0,73 \pm 0,119$ & $1,24 \pm 0,155$ & 40,32 & 1,46 & 6,5 & $\mathrm{~m}$ \\
\hline 6 & $0,55 \pm 0,020$ & $0,67 \pm 0,048$ & $1,22 \pm 0,062$ & 45,08 & 1,21 & 6,5 & $\mathrm{~m}$ \\
\hline 7 & $0,48 \pm 0,034$ & $1,24 \pm 0,163$ & $1,72 \pm 0,148$ & 27,90 & 2,58 & 10,9 & $\mathrm{sm}$ \\
\hline 8 & $0,52 \pm 0,079$ & $1,00 \pm 0,171$ & $1,53 \pm 0,223$ & 33,98 & 1,92 & 8,2 & $\mathrm{sm}$ \\
\hline 9 & $0,48 \pm 0,067$ & $0,93 \pm 0,110$ & $1,41 \pm 0,177$ & 34,04 & 1,93 & 8 & $\mathrm{sm}$ \\
\hline 10 & $0,48 \pm 0,034$ & $0,89 \pm 0,134$ & $1,38 \pm 0,115$ & 34,78 & 1,85 & 7,4 & $\mathrm{sm}$ \\
\hline 11 & $0,47 \pm 0,043$ & $0,85 \pm 0,098$ & $1,32 \pm 0,098$ & 35,60 & 1,80 & 7 & $\mathrm{sm}$ \\
\hline 12 & $0,44 \pm 0,050$ & $0,86 \pm 0,083$ & $1,30 \pm 0,133$ & 33,84 & 1,95 & 7 & $\mathrm{sm}$ \\
\hline 13 & $0,43 \pm 0,031$ & $0,84 \pm 0,070$ & $1,27 \pm 0,062$ & 33,85 & 1,95 & 6,8 & $\mathrm{sm}$ \\
\hline
\end{tabular}


Tabla 3. Datos morfométricos de los cariotipos de A. colubrina var. cebil y $P$. rigida.

(1) Longitud cromosómica media \pm error estándar; (2) índice centromérico medio \pm error estándar; (3) razón 1/s medio ; (4) proporción entre el par cromosómico mayor y menor del complemento y (5) longitud cromosómica total.

\begin{tabular}{lcc}
\hline Datos & A. colubrina & P. rigida \\
\hline $2 \mathrm{n}$ & 26 & 26 \\
$x$ & 13 & 13 \\
Fórmula del cariotipo & $18 \mathrm{~m}+8 \mathrm{sm}$ & $12 \mathrm{~m}+14 \mathrm{sm}$ \\
Satélites & par 13 & par 9 \\
LCT en $\mu$ m $(5)$ & 38,88 & 36,66 \\
c $(\mu \mathrm{m}) \pm \mathrm{ES}(1)$ & $1,49 \pm 0,086$ & $1,41 \pm 0,042$ \\
i \pm ES $(2)$ & $40,33 \pm 0,013$ & $37,58 \pm 0,013$ \\
r土 ES $(3)$ & $1,50 \pm 0,088$ & $1,69 \pm 0,103$ \\
R (4) & 2,161 & 1,409 \\
A1 & 0,306 & 0,382 \\
A2 & 0,210 & 0,107 \\
Tipo de asimetría de Stebbins & B2 & $\mathrm{A} 1$ \\
\hline
\end{tabular}

puesto que A. colubrina tiene 38,88 micras y $P$. rigida 36,66 micras (Tabla 3 ).

El grado de asimetría intracromosómica (A1) es mayor en P. rigida por la variación en las relaciones de brazos, mientras que las variaciones intercromosómicas (A2) son mayores en $A$. colubrina, indicando mayor variación en las longitudes cromosómicas (Tabla 3). Los cariotipos de ambas especies presentan valores de asimetría cercanos entre sí. Por otro lado, de acuerdo con las categorías de Stebbins (1971) A. colubrina (tipo B2) tendría un cariotipo más asimétrico que $P$. rigida (tipo A1).

Citológicamente ambas especies son idénticas en cuanto al número de cromosomas. Las diferencias se encuentran en la fórmula cariotípica, el tamaño de los cromosomas, la posición del satélite y la longitud total del complemento cromosómico, lo que apoyaría su separación taxonómica actual.

\section{Agradecimieritos}

Queremos expresar nuestro agradecimiento a la Lic. A. Cardozo quién nos facilitó parte del material y colaboró en la confección de los preparados y al Lic. E. Permingeat por la confección de los idiogramas.

\section{Bibliografía}

ALTSCHUL, S. 1964. A taxonomic study of the genus Anadenanthera. Contr. Gray Herb. 193:3-65.
BRENAN, J.P.M. 1955. Notes on Mimosoideae: I. Kew Bull. 10 (2): 161-183

1963. Notes on Mimosoideae: VIII. Kew Bull. 17 (2):227228.

BURKART, A. 1943 Leguminosas argentinas, silvestres y cultivadas. Ed. ACME. 588 págs.

1949. Leguminosas nuevas o críticas, III. Darrviniana 9 (1):63-96.

1969. Leguminosas nuevas o críticas, VII. 3. La subdivisión del género Piptadenia Benth. sensu lato. Darwiniana 15 (3-4): 504-506.

1972. Leguminosas, en DIMITRI M., Enciclopedia de Agricultura y Jardinería. Tomo I. Ed. ACME, 1028 págs.

CASTIGLIONI, J.A.1979. Descripción botánica, forestal y tecnológica de las principales especies indígenas de la Argentina. En D.Cozzoct al. Arboles forestales, maderas y silvicultura de la Argentina, Enciclopedia Arg. Agric. y Jardinería. Fasc. 16-1. Ed. ACME.

COWAN, R.S. \& j.P.M. BRENAN .1960. Typification of Piptadenia Bentham. Taxon 9:56.

DIGILIO, A.P.L. y P. LEGNAME. 1966. Los árboles indígenas de ia provincia de Tucumán. Opera Lilloana 15.

GIBBS P.E. \& R. INGRAM. 1982. Chromosome numbers of some brazilian flowering plants. Notes Roy. Bot.Gard. Edinburgh 40:399-407.

GOLDBLATT, P. 1981. Chromosome Numbers in Legumes II. Ann. Missouri Bot. Gard. 68: 551 - 557.

HUNZIKER, A.T. 1973. El cebil (Anadenanthera colubrina var. cebil) en la Provincia de Córdoba. Kurtziana 7:265

LEVAN, A., K. FREDGA \& A.A. SANDBERG. 1964. Nomenclature for centromeric position on chromosomes. Hereditas 52: 201-220.

ROMERO ZARCO, C. 1986. A new method for estimating karyotype asymmetry. Taxon $5: 526-530$.

SPEGAZZINI, C. 1922. Algunas observaciones relativas ai suborden de las Mimosoideas. Physis (Buenos Aires) 6:308-315.

STEBBINS, G.L. 1971. Chromosomal evolution in higher plants. E. Arnold. London. 216 págs. 